Ciencias Ambientales/Environmental Sciences

Cienc Tecn UTEQ (2018) 11(1) p 37-43 ISSN 1390-4051; e-ISSN 1390-4043

\title{
Distribución espacial de Elasmobranquios en la costa continental ecuatoriana
}

\author{
Spatial distribution of Elasmobranchs on the Ecuadorian continental coast \\ Moreno-Vera, Ana ; Díaz-Ponce, Mariela ${ }^{1}$; Acosta-Farías, Milena ${ }^{2}$; Jimenez, Edwin ${ }^{1}$; Saltos, Jorge ${ }^{3}$; Neira, Jorge ${ }^{3}$ \\ ${ }^{1}$ Facultad de Ciencias Ambientales, Universidad Técnica Estatal de Quevedo; Campus Manuel Haz Álvarez, Av. Quito km. 1 1/2 \\ vía a Santo Domingo de los Tsáchilas, EC-120501. Quevedo, Ecuador \\ ${ }^{2}$ Área de Ciencias de la Vida, Universidad Politécnica Salesiana; Campus Girón, Av. Isabel La Católica N. 23-52 y Madrid. \\ Quito, Ecuador \\ ${ }^{3}$ Gobierno Autónomo Descentralizado Municipal de Quevedo. Quevedo, Ecuador.
}

Autora para correspondencia: amoreno@uteq.edu.ec

Rec.: 27.02.2018. Acept.: 31.05.2018 Publicado el 2 de Julio de 2018

\section{Resumen}

$\mathrm{D}$ urante siglos, los pescadores practicaron de manera sostenible la pesca de tiburón en aguas costeras del Ecuador; aunque se dispone de poca información en cuanto a las capturas y estado de las especies, la presente investigación se planteó como objetivos determinar, a través del cálculo de la riqueza ecológica, las especies distribuidas en un área que abarca desde el norte de Manta en la provincia de Manabí $(00059.92$ 'S - 80053.93'W) hasta la frontera sur del Ecuador $\left(03^{\circ} 23.10^{\prime} \mathrm{S}-80027.60^{\prime} \mathrm{W}\right)$ y su relación espacial entre las capturas, mediante cruceros oceanográficos desde noviembre de 1994 hasta julio de 1999, utilizando redes de arrastre camaroneros en un recorrido por tres estratos de profundidad; el estrato I (10 a 30 m), el II (31 a 80 m) y el III (81 a 200m); cuyo fin fue determinar las variables de volúmenes de capturas y la incidencia de las redes de arrastre, como uno de los mayores problemas medioambientales que puede provocar afectaciones a la estructura y funcionamiento de los sistemas marinos y a sus poblaciones, a nivel de comunidades y de ecosistemas, agravadas por el incumplimiento de las medidas de control y ordenamiento. Los volúmenes de pesca por estratos, el Golfo de Guayaquil, fue el sitio con mayor número de especies recolectadas por los cruceros de exploración. Estos datos servirán de base para la elaboración y soporte del plan nacional de manejo y conservación de tiburones para aguas ecuatorianas.

Palabras clave: Riqueza específica, estratos de pesca, pesca incidental
$\mathrm{F}$ or centuries, fishermen have practiced shark fishing in the coastal waters of Ecuador; although little information is available on catches and species status. The present research aimed to determine, through the calculation of ecological wealth, the species distributed in an area that covers from the north of Manta in the province of Manabí (00059.92'S $\left.80053.93^{\prime} \mathrm{W}\right)$ to the southern border of Ecuador $\left(03^{\circ} 23.10^{\prime} \mathrm{S}\right.$ - 80027.60'W) and the spatial relationship between catches, by oceanographic cruisers from November 1994 to July 1999, using networks of drag shrimp trawlers on a course of three depths; stratum I (10 to $30 \mathrm{~m})$, II (31 to $80 \mathrm{~m}$ ) and III (81 to $200 \mathrm{~m}$ ); whose purpose was to determine the variables of catch volumes and the incidence of trawl nets as one of the major environmental problems that can affect the structure and functioning of marine systems and their populations at the community and ecosystem levels, aggravated by noncompliance with control and management measures. In terms of fishing volumes by strata, the Gulf of Guayaquil, was the site with the highest number of species collected by exploration cruisers. These data will serve as a basis for the elaboration and support of the national shark management and conservation plan for Ecuadorian waters.

Keywords: specific wealth, fishing strata, by-catch 


\section{Introducción}

$\mathrm{E}$ muchas partes del mundo, el tiburón es considerado como una fuente importante de proteínas, de empleo y de beneficios económicos para quienes se dedican a su pesca, comercialización y consumo.

Existen recursos genéticos del mar que son explotados de manera indiscriminada, tal es el caso de las diferentes especies de tiburones, los mismos que son capturados para utilizar en la mayoría de los casos únicamente las aletas, sin aprovechar el resto del cuerpo. En función de esta realidad, en algunos países, ya existen evidencias de una tendencia hacia la exportación de las alteas y el consumo nacional de la carne de tiburón. Por lo tanto, los principales problemas a nivel mundial son: el aleteo y el desperdicio de un recurso alimenticio.

En Ecuador se han reportado 23 familias de elasmobranquios, agrupados en 46 especies de tiburones y 22 de rayas, guitarras y torpedos. La existencia de elasmobranquios está amenazada debido al aumento de la demanda de su carne y partes a nivel internacional, sumado a esto las prácticas antitécnicas de pesca como el arrastre y palangre que aumenta significativamente la captura incidental.

Entre las especies reportadas en los desembarques industriales y artesanales están aquellas pertenecientes a las familias Alopiidae, Carcharhinidae, Lamnidae, Sphyrnidae y Triakidae en el grupo de los tiburones; mientras que en el grupo de las mantas y rayas están especies de las familias Dasyatidae, Molubidae, Myliobatidae, Rajidae y Rhinopteridae. La mayoría de los elasmobranquios que han sido registrados en aguas ecuatorianas son especies transzonales o altamente migratorias.

Coello (2005) registra nuevas especies de elasmobranquios: Megachasma pelagios y Carcharhinus signatus. Respecto a M. pelagios, los avistamientos pueden tener alto grado de incertidumbre debido a los escasos registros mundiales de este organismo. De igual forma, Coello reconoce que existen otros registros de tiburones, pero no han sido publicados, por lo que el número total de especies presentes en Ecuador seguramente es mayor.

En aguas ecuatorianas existen 31 elasmobranquios incluidos en la listan roja de especies amenazadas de la Unión Internacional para la Conservación de la Naturaleza (UICN, 2011), de las cuales cuatro de ellas son consideradas vulnerables. La categoría "vulnerable" pone a estas especies en el plano de la conservación y las medidas de protección deberán ser de estricta responsabilidad de la Subsecretaria de Recursos Pesqueros y del Ministerio del Ambiente (Plan de Acción Nacional para la Conservación y Ordenación de Tiburones en el Ecuador, 2006).

Según estudios realizados en el 2005 por Coello (2005), quien manifiesta que la pesca de rayas en Ecuador se ha iniciado hace tres años y al igual que en el resto del mundo, se ha incrementado, y se ha convertido en una pesca dirigida por la alta demanda de su carne (Homma, et al. 1999, Bearez
2004, Larson et al. 2004, Baquero 2006) y por sus espinas que son comercializadas como artesanías (Stevens et al. 2000, Denkinger). Esta creciente demanda ha colocado a la raya coluda (Dasyatis longa), entre los cinco géneros de elasmobranquios más capturados en el Ecuador, junto al tiburón ángel (Squatina californica), tiburón martillo (Sphyrna zygaena), tiburón come perro (Carcharhinus leucas) y tiburón zorro (Alopias spp.).

Durante la revisión de información bibliográfica, respecto a trabajos que hayan permitido determinar el impacto de redes de arrastre sobre los elasmobranquios demersales, fue imposible encontrar registros anteriores que permitan ampliar el conocimiento de la biología pesquera de dichos organismos (Coello, 2005).

El manejo de la pesquería en Ecuador presenta falencias en el control y la implementación de regulaciones, por tanto, no existe información relevante relacionada a la distribución, abundancia, biología ni procesos críticos de la vida de tiburones como épocas y zonas de apareamiento, hábitats críticos, con lo que resulta difícil evaluar el estado de las poblaciones en aguas ecuatorianas.

La explotación incidental de tiburón en aguas ecuatorianas tiene una mediana y alta representatividad en su captura, debido a que es realizada desde diversas fuentes propiciadas por los diferentes tipos de pesquerías, las artes de pesca y las especies de elasmobranquios estudiadas en este trabajo, ocasionando de manera directa su alteración biológica, ecológica, desarrollo y distribución. Razón por la cual, su estudio debe hacerse de forma urgente (Guerrero, 2001).

Realmente se dispone de poca información en cuanto a las capturas o la estimación de sus desembarques. Posiblemente uno de los primeros trabajos publicados sobre este tema es de Herdson et al. (1985), en donde se hace referencia a los volúmenes de tiburón desembarcados en el año de 1982 (Martínez, 2006).

La información obtenida de las capturas de Condrictios, durante los cruceros demersales, fue analizada para determinar la distribución espacial de elasmobranquios representativos, riqueza biológica de las especies de condrictios a diferentes estratos de profundidad y respecto a los volúmenes de captura.

\section{Materiales y métodos}

\section{Área de estudio}

El área está ubicada en la plataforma continental de la costa ecuatoriana, comprendida desde Manta en la provincia de Manabí (00059.92'S - 80053.93’W) hasta la frontera sur de Ecuador $\left(03^{\circ} 23.10^{\prime} \mathrm{S}-80027.60^{\prime} \mathrm{W}\right)$ en los límites de la frontera marina con Perú, que abarcan los 12 metros cerca de la línea de costa y 128 metros de profundidad. Mediante cruceros de exploración ejecutados por el Instituto Nacional de Pesca (INP) utilizando red de arrastre, fueron ejecutados desde noviembre de 1994 hasta julio de 1999 y que comprenden $89228,50 \mathrm{~km} 2$ son el objeto de estudio de esta investigación. 
Recolección y análisis de datos

Los datos analizados para la investigación fueron tomados de boletines científicos que contienen resultados de lances de pesca por estratos de profundidad de los cruceros demersales (Cuadro 1) efectuados en la costa ecuatoriana entre 1994 y 1999.

Cuadro 1. Cruceros de pesca demersales realizados por el Instituto Nacional de Pesca desde noviembre de 1994 a julio de 1999

\begin{tabular}{|c|c|c|c|c|c|c|c|}
\hline \multirow[b]{2}{*}{$\mathbf{N}^{\mathbf{o}}$} & \multirow[b]{2}{*}{ Cruceros } & \multirow[b]{2}{*}{ Fechas } & \multirow[b]{2}{*}{ Referencias } & \multicolumn{4}{|c|}{ Número de lances por estratos } \\
\hline & & & & Estrato 1 & Estrato 2 & Estrato 3 & $\begin{array}{c}\text { Lances } \\
\text { totales }\end{array}$ \\
\hline & & & & $(10-30 \mathrm{~m})$ & $(31-80 \mathrm{~m})$ & $(81-200 \mathrm{~m})$ & \\
\hline 1 & T94/11/18 D & Noviembre 1994 & Revelo (1994) & 8 & 17 & 5 & 30 \\
\hline 2 & T95/06/21 D & Junio 1995 & Revelo (1995) & 10 & 0 & 0 & 10 \\
\hline 3 & T96/05/24 D & Мауо 1996 & Revelo et al. (1996) & 11 & 51 & 12 & 74 \\
\hline 4 & T98/07/03 D & Julio 1998 & Revelo (1998) & 15 & 31 & 5 & 51 \\
\hline 5 & T98/10/05 D & Octubre 1998 & Revelo et al. (1998) & 15 & 35 & 6 & 56 \\
\hline 6 & T99/07/02 D & Julio 1999 & Herrera et al. (2001) & 14 & 44 & 14 & 72 \\
\hline
\end{tabular}

Para la toma de muestra en estos estudios, se utilizó una red de arrastre de fondo modelo italiano GOV 35/42 que tiene la forma de una bolsa cónica, con una boca ancha rodeada de flotadores montados en una cadena de $3 / 8$ ", con dimensiones de $33 \mathrm{~m}$ de relinga superior y $32,2 \mathrm{~m}$ de relinga inferior; cuya función fue barrer el fondo del mar-específicamente en sitios identificados mediante ecosonda, que permite estimar la profundidad y las condiciones del fondo- y capturar la mayor cantidad de peces posibles, los mismos que quedan retenidos dentro de la red para su posterior identificación. Los lances tuvieron una duración entre 15 y 33 min de arrastre efectivo. Para la identificación de las especies capturadas se procedió a pesar el volumen de los peces clasificados como comestibles y no comestibles y posteriormente se ubicaron por categorías taxonómicas (Ebert, D. y Mostarda, E., 2016).

\section{Especies seleccionadas}

En el Cuadro 2 se muestran las familias y las especies analizadas en el presente estudio, las mismas que fueron seleccionadas con base en los reportes generados a partir de los cruceros oceanográficos publicados por el Instituto Nacional de Pesca, teniendo como principal criterio las especies mayormente capturadas.

Cuadro 2. Especies seleccionadas para el análisis de riqueza ecológica

\begin{tabular}{lll}
\hline Familias & Nombre Científico & Nombre Vulgar \\
\hline Squatinidae & Squatina armata & angelote \\
Triakidae & Mustelus sp. & tollo \\
Heterodontidae & Heterodontus quoyi & tiburón gato \\
Rhinobatidae & Zapterix exasperata & guitarra \\
Rajidae & Raja equatorialis & raya \\
& Raja velezii & raya \\
\hline
\end{tabular}

\section{Análisis de riqueza ecológica}

El cálculo de la riqueza ecológica de los elasmobranquios, fue realizado por especies y por zonas de pesca, con la finalidad de determinar su presencia o ausencia, usando para ello la siguiente fórmula:

\section{Donde:}

$$
\mathrm{R}=\sum \mathrm{X}_{\mathrm{i}, \mathrm{j}}
$$

$\mathrm{R}=$ riqueza ecológica

$\mathrm{X}=$ presencia o ausencia

$\mathrm{i}=$ especie

$\mathrm{j}=$ zona de pesca
Seguidamente, se utilizó el programa Surfer versión 6.01 para elaborar los mapas de distribución de capturas totales de elasmobranquios expresados en kilogramos, por número de lances a diferentes estratos de profundidad. Dentro del programa se seleccionó la opción de tratamiento de los datos por "Krigin, (Petitgas y Rivoirard, 1991), y "distancia inversa (Pennington, 1983), de geoestadística (Rossi, et al., 1992).

Adicionalmente, se realizó un análisis estadístico descriptivo donde se muestra por estratos de profundidad y por especies la distribución y presencia de los tipos de individuos capturados. 


\section{Resultados y discusión}

$\mathrm{D}$ istribución espacial de principales especies de tiburones demersales

El área cubierta por los cruceros demersales realizados por el B/I Toallí en la costa continental ecuatoriana, por lances y por estratos se exponen en el Cuadro 3. Donde el estrato II (31-80 $\mathrm{m}$ de profundidad) es el más abundante en número de especies, seguido del estrato I y en menor proporción se encuentra el estrato III.

Estudios con perfiles que consiguen determinar la estructura espacial de los recursos de elasmobranquios demersales toman relevancia en zonas de importancia social, económica y sistémica, más aún con la tendencia de visión ecológica de los sistemas pesqueros tropicales, (Wenner y Sedberry,
1989). Durante el análisis goestadístico (Rossi, et al, 1992) y de geo-referenciación de las capturas experimentales de elasmobranquios demersales, fueron empleados los datos de 293 lances de pesca de arrastre de fondo realizados en 6 cruceros de investigación del B/I Tohallí, entre 1994 y 1999. En términos generales, los resultados provistos por el INP respecto a estos cruceros han sido asociados a la estimación de densidades y biomasa total de las principales especies de peces comerciales a partir del método de área barrida (Herrera, et al., 1999); sin embargo, en todos los informes de cruceros de investigación que han realizado capturas con red de arrastre de fondo, hasta ahora no se ha reportado la relación de las capturas que utiliza dicho arte de pesca, respecto al impacto sobre la fauna de tiburones, rayas y torpedos que habitan en los fondos.

Cuadro 3. Áreas cubiertas por los cruceros demersales y lances de pescas realizados por estratos

\begin{tabular}{lrrr}
\hline & Superficie Km2 & Lances de pesca & Individuos capturados (tiburones y rayas) \\
\hline Estrato I & 35420.30 & 111 & 498 \\
Estrato II & 32761.50 & 405 & 2377 \\
Estrato III & 21106.70 & 117 & 1093 \\
Total & 89288.50 & 633 & 3968 \\
\hline
\end{tabular}

En cuanto a la distribución espacial de las principales especies de tiburones demersales provenientes de las capturas realizadas en los diferentes estratos de profundidad se aprecian mayores concentraciones de elasmobranquios a lo largo del perfil costanero en el estrato II sin embargo, en los estratos I y III las concentraciones decaen. En la plataforma continental desde la provincia de Esmeraldas hasta la provincia de El Oro, se han registrado capturas de elasmobranquios demersales en los cruceros de investigación, siendo los sitios con mayor presencia las provincias de Guayas y El Oro (Golfo de Guayaquil) y Santa Elena (hacia los dos frentes, norte y sur, de la Puntilla de Santa Elena) (Figura 1).

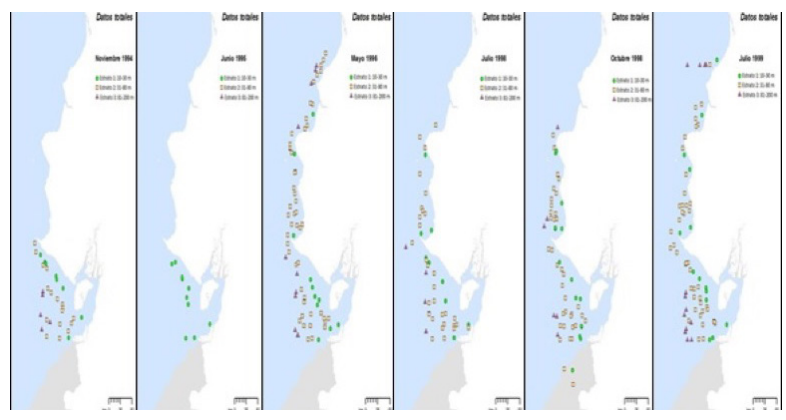

Figura 1. Distribución espacial de las capturas totales de elasmobranquios por lances y por estratos durante los meses de noviembre de 1994, junio 1996, mayo 1996, julio 1998, octubre 1998 y julio 1999 (INP, 1994-1999)

Mustellus spp., es la especie con mayor distribución en toda la costa del Ecuador, mientras que Heterodontus quoyi tuvo una distribución restringida hacia el Golfo de Guayaquil. En cuanto a las rayas, tanto la $R$. velezii como $R$. equatorialis tuvieron distribuciones desde la provincia de Manabí hacia el Golfo de Guayaquil. La especie que registró la distribución más al sur del país, en la frontera con el Perú fue Squatina armata.

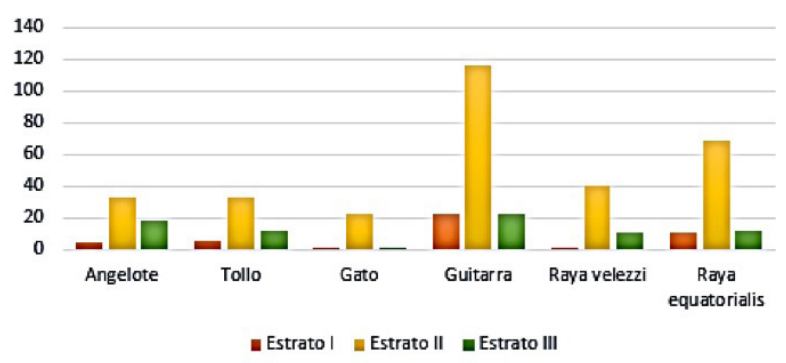

Figura 2. Número de individuos recolectados por pesca de arrastre en diferentes estratos.

Cabe mencionar que en la costa continental de Ecuador opera una flota industrial conformada por 156 embarcaciones de madera que usan como arte de pesca la red de arrastre para la captura de camarón marino. Dicha flota realiza sus actividades de pesca en sitios tales como el Golfo de Guayaquil y los frentes Norte y Sur de la Puntilla de Santa Elena (Figs. 3 y 4), sitios que coinciden con los mayores lugares de distribución y captura incidental de elasmobranquios registrados en las operaciones de pesca científica desde el B/I Tohallí. 


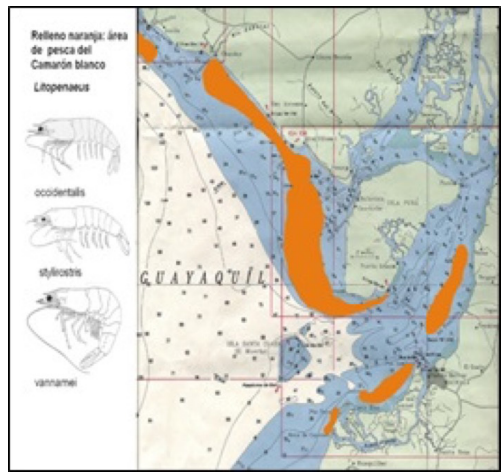

Figura 3. Áreas de pesca del camarón blanco del género Penaeus reportados por la Asociación Ecuatoriana de Armadores de Barcos Pesqueros Camaroneros: Provincia de Santa Elena y Manabí (ASAERBAPESCA, 2002)

Por otra parte, el estado ecuatoriano a través de la Subsecretaría de Recursos Pesqueros, en un esfuerzo por regular las actividades de la flota arrastrera camaronera, en noviembre de 2009, emite el Acuerdo Ministerial 162 donde entre otros temas, incluye una zonificación de las áreas de pesca de dicha flota; en el que muchos de los sitios enunciados en dicho Acuerdo coinciden con las áreas donde los elasmobranquios demersales se distribuyen, (MAGAP, 2009)

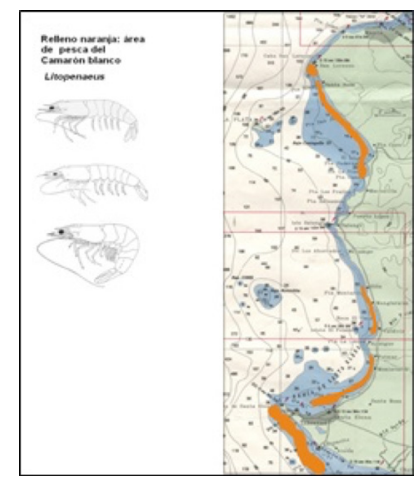

Figura 4. Áreas de pesca del camarón blanco del género Penaeus reportados por la Asociación Ecuatoriana de Armadores de Barcos Pesqueros Camaroneros: Golfo de Guayaquil (ASAERBAPESCA, 2002).

\section{Riqueza ecológica}

La mayor riqueza ecológica se observó en el estrato II y la menor proporción de especies capturadas se definió en el estrato III (Fig. 5). Cabe mencionar, que las especies de tiburones Squatina armata (angelote), Mustelus sp. (tollo) y Heterodontus quoyi (gato) y las rayas Zapterix exasperata (guitarra), Raja Velezii (raya) y Raja equatorialis (raya) son las especies que mostraron mayor incidencia a la captura por red experimental de arrastre de fondo según el estrato de profundidad.

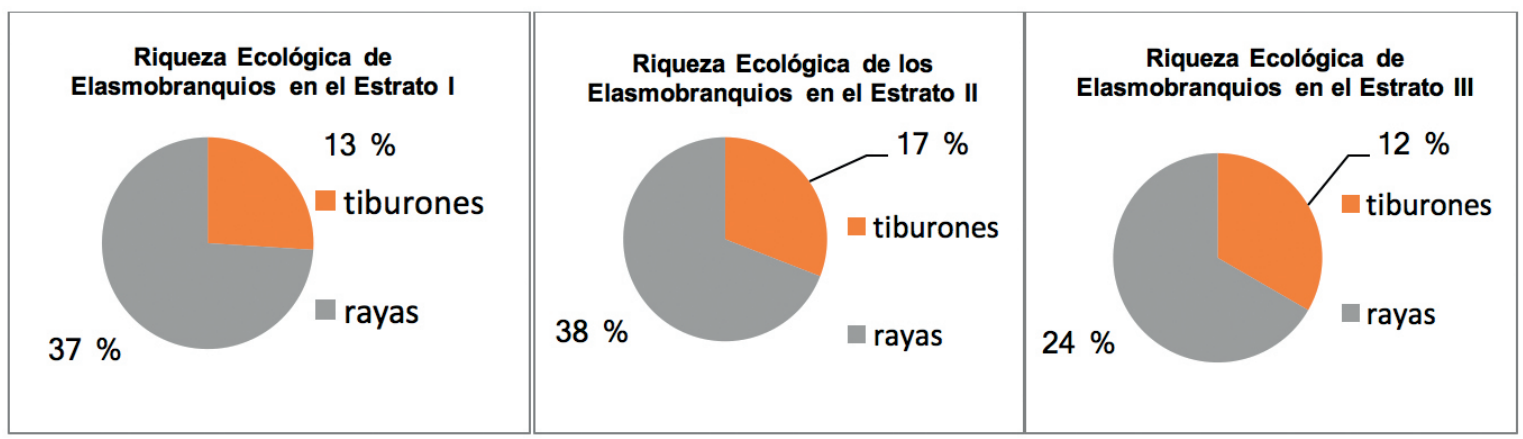

Figura 5. Relaciones porcentuales de elasmobranquios a diferentes estratos de profundidad. A) Riqueza ecológica de Elasmobranquios en el estrato I. B) estrato II. C) estrato III

Los resultados del análisis de distribución de elasmobranquios por estratos de profundidad, confronta la discusión sobre conservación versus explotación, nuevamente asociada con la operación de flotas pesqueras; especialmente de la flota arrastrera camaronera.

La pesquería de arrastre de camarón opera desde la década de 1950 en los fondos blandos de la plataforma continental y tiene un alto componente de pesca acompañante. Little \& Herrera (1992) registraron 261 especies en la pesca acompañante en el año 1991. Arriaga \& Martínez (2002) señalan 217 especies de peces y unas 40 mil toneladas anuales que son retenidas $\mathrm{y}$ aprovechadas para consumo interno y de exportación. Los tiburones y rayas, principalmente especies pequeñas y costeras, siempre han sido un elemento constitutivo de la captura incidental.

Las rayas han representado históricamente un alto porcentaje de la captura. Little \& Herrera (1992) estimaron que las rayas constituyeron, respectivamente, $31 \%$, 38\% y $50 \%$ de la captura (en peso) de los barcos langostineros al norte, centro y sur de la plataforma continental en el año 1991. Estos autores también reportan que las rayas constituyeron $33 \%$ de la captura de los barcos pomaderos en el mismo año (1992). En esos años las rayas eran casi en su totalidad descartadas. Históricamente sólo los tiburones grandes han sido retenidos. Herrera et al (2003), reportan 11 especies de tiburones en la captura incidental de los barcos arrastreros. 
Estos mismos autores reportaron que tiburones de la familia Triakidae estuvieron presentes en entre $10 \%$ y $22 \%$ de los lances de los barcos langostineros; mientras que tiburones de la familia Sphyrnidae estuvieron presente en entre 1\% y 9\% de los lances. Estos autores también reportaron que en los barcos pomaderos la familia Triakidae estuvo presente en $6 \%$ de los lances, y la familia Sphyrnidae en menos $0.5 \%$ de los lances. La preservación (i.e., proteger y resguardar estrictamente ambientes y especies frágiles ó esenciales) es también una opción que no ha sido suficientemente analizada. Desde que la conservación y preservación de especies ha tomado fuerza, se desarrollan campañas que apelan a los sentimientos humanos, pero no se proveen análisis que sustenten los costos y beneficios (entendiendo que muchos de estos son intangibles) de la opción de no usar los tiburones. Vale destacar que, si bien los tiburones han sido adoptados como especie emblema de la conservación marina, las rayas -- que probablemente enfrentan severos problemas de conservación, por ejemplo, han constituido por décadas un alto porcentaje de las capturas de los barcos arrastreros de camarón (Little \& Herrera, 1992) -- han sido totalmente excluidas de estas iniciativas.

Seis especies de elasmobranquios fueron dominantes en el área de estudio, por su abundancia y distribución están relacionadas con las diferentes estrategias biológicas para utilizar los hábitats del sistema con fines alimenticios, reproductivos o de protección, como también con las variaciones ambientales (temperatura, salinidad, transparencia, profundidad) del sistema a lo largo del tiempo (Horn y Allen 1985, YáñezArancibia et al., 1988), por tanto, son variables importantes en la distribución y abundancia de las especies dominantes en la comunidad de tiburones y rayas.

Así, la composición de las especies de condrictios, respecto a la relación distribución espacial de las capturas-estrato de profundidad, se asumen relaciones de distribución espacial diferenciadas, donde los estratos de profundidad II (31-80 m) y III (81-300 m) fueron los que resultaron con diferencias $(p<0.05)$ para tres especies de rayas.

Aunque estos resultados no son determinantes, ya que respuestas a fijar la distribución espacial de organismos demersales requieren de otras variables ambientales tales como temperatura, salinidad y tipos de sustrato; hasta ahora con estos primeros resultados se puede asumir que profundidades entre 80 y $300 \mathrm{~m}$ están directamente relacionadas con la distribución de las rayas: Raja equatorialis, $R$. velezi $\mathrm{y}$ Zapterix exasperata.

Contrario a los resultados para rayas, las 3 especies de tiburones con predominancia en las capturas: Heterodontus quoyi, Mustelus spp., y Squatina armata, no presentaron diferencias de distribución por estratos de profundidad. El hecho de que dichas especies de tiburones no presenten claras relaciones de distribución respecto a la profundidad puede estar asociado que dichos elasmobranquios tienen comportamiento migratorio, lo que a su vez aumenta su rango de distribución, tanto en los niveles de profundidad (desplazamiento vertical), como en su movimiento hacia otras regiones geográficas (desplazamiento horizontal).

La mayor o menor incidencia de las capturas de elasmobranquios pueden estar asociadas a variables oceanográficas, especialmente corrientes marinas que surgen en nuestro país, como la corriente fría de Humbolt que produce aumento en cuanto a la productividad y es favorable para la pesca de recursos demersales (julioseptiembre) y el Evento del Niño o ENOS donde la productividad sufre un descenso y las pesquerías del recurso demersal decaen (diciembre-abril) (Revelo, De la Cuadra, Macías et al, 1998; Herrera et al., 1999; Gerrón, 2007).

Es posible que el presente documento sea el primer trabajo realizado para determinar la incidencia de redes de arrastre demersal sobre elasmobranquios demersales, considerándose inédito, siendo éste, la base para futuros trabajos que permitan ampliar el conocimiento sobre las implicaciones que tiene el uso de redes de arrastre demersal en la diversidad de tiburones y rayas demersales, así como de trabajos que hagan énfasis en la biología pesquera de al menos las principales especies de tiburones y rayas demersales que puedan ser afectados por artes de pesca no selectivos en ecosistemas tropicales.

\section{Conclusiones}

os recursos demersales de la plataforma continental de la costa ecuatoriana, desde Esmeraldas en el Norte del país (latitud $01000^{\prime} \mathrm{N}$ ) hasta la frontera sur del Ecuador (latitud 03023 S), siguen un patrón específico de distribución, el mismo que está relacionado con los estratos de profundidad en los que dichos recursos se distribuyen, de acuerdo a la data evaluada entre 1994 y 1999.

Estos estratos de profundidad poseen características físicas relevantes (i.e., tipo de sustrato, fondos irregulares, distancia a la costa) que interactúan entre si, y que confiere a cada estrato la particularidad de alojar a los grupos taxonómicos de ambiente marino dentro de uno o varios estratos, los mismo que pueden desarrollar sus características bioecológicos tales como reproducción, alimentación y protección, dentro de hábitats definidos.

En este sentido, la mayor abundancia de especies demersales se encontraron distribuidas dentro de los estratos II (31-80 m) y III (81-300), los mismos que poseen diversificación en cuanto a los tipos de sustrato localizados en toda la línea de costa ecuatoriana. Hacia el norte y centro del estrato II existen fondos blandos caracterizados por depósitos de arena y lodo, mientras que hacia el sur existen fondos rocosos e irregulares. El sitio de mayor importancia para la distribución de los elasmobranquios demersales fue el golfo de Guayaquil, desde el centro del Golfo hacia el Sur del país en frontera con el Perú. La red de arrastre demersal tiene gran incidencia en la captura de elasmobranquios demersales, principalmente de las especies de tiburones angelote (Squatina armata), tollo (Mustellus sp.) y tiburón gato (Heterodontus quoyi); y entre las rayas Raja velezii, Raja equatorialis y guitarra (Zapterix 
exasperata).

\section{Bibliografía}

Aguilar, F., Chalén, X., Villón, C. (2005). Plan de Acción Nacional para la Conservación y Ordenación de los Tiburones (PAT-Ecuador). Instituto Nacional de Pesca.

ASEARBAPESCA. (2002). Caracterización de la flota arrastrera camaronera y la problemática que implicaría establecer una zonificación. ASEARBAPESCA Documento Técnico. 44 pp. Guayaquil, Ecuador.

Herrera, M., Solís - Coello, P., Vicuña, H., Macias, P., Coello, D., Moya, O., Luzuriaga, M. y Elías, E. (2001). Estimación de la Biomasa de los Recursos Demersales en la Plataforma Continental del Ecuador durante julio de 1999. Instituto Nacional de Pesca. Boletín Científico Técnico. Vol. XVII. 112 pp. Guayaquil - Ecuador.

Revelo, W., Herrera, M., De la Cuadra, T., Macías, P., Coello, D., Moya, O., Elías, E., Ortega, D. y Vicuña, H. (1998). Estimación de la Biomasa de los Recursos Demersales en la Plataforma Continental del Ecuador durante octubre de 1998. Instituto Nacional de Pesca. Boletín Científico Técnico. 112 pp. Guayaquil - Ecuador.

Herrera, M., Revelo, W., De la Cuadra, T., Macías, P., Coello, D., Moya, O., Elías, E., Ortega, D. y Vicuña, H. (1998). Estimación de la Biomasa de los Recursos Demersales en la Plataforma Continental del Ecuador durante octubre de 1998. Instituto Nacional de Pesca. Boletín Científico Técnico. 112 pp. Guayaquil - Ecuador.

Horn, MH., \& Allen, LG. (1985). Fish community ecology in southern California bays and estuaries. pp. 169- 190. In A. Yáñez-Arancibia (ed.). Fish Community Ecology in Estuaries and Coastal Lagoons: Towards an Ecosystem Integration. Universidad Nacional Autónoma de México. México.

Revelo. W, J. González y H. Vicuña. (Mayo 1996). Evaluación de Recursos Demersales en la Plataforma Continental de Ecuador, Vol. XIV, No 5, Boletín Científico y Técnico, pp. 40-67, I.N.P.

Little, M., y Herrera, M. (1992). The by-catch of the Ecuadorian shrimp fleet. 1991. Internal, Report. INP-ODA. 90 p.

MAGAP. 2009. Acuerdo Ministerial No. 162. Medidas de ordenamiento, regulación, control, zonificación e investigación de la flota arrastrera ecuatoriana.

Oviedo, M. (2010). La Pesca Artesanal, el Acceso a los Recursos, Sistemas de Manejo y Zonas de Pesca: el caso de Ecuador y las Islas Galápagos. Coordinador. Proyecto Araucaria de la Agencia Española de Cooperación Internacional AEC. (http://www.icsf.net/icsf2006/ jspFiles/cedepesca/presentaciones/oviedo_galapagos/ oviedo galapagos acta.htm).

Pennington, M. (1983). Efficient estimators of abundance, for fish and plankton survey, Biometric, 39:.281-286 pp.

Petitgas, P. y Rivoirard, J. (1991). Global estimations: $\sigma 2 / \mathrm{n}$ and the geostatistical estimation variance, Appendix B in Anon. 1991, CM91/D: 40 pp.

ONU. (Organización de las Naciones Unidas para la Agricultura y la Alimentación). (1999). Plan de Acción Internacional para la Conservación y Ordenación de los Tiburones. Roma. 23-24 pp.

Revelo, W., González y Vicuña, H. (mayo, 1996). Evaluación de Recursos Demersales en la Plataforma Continental de Ecuador. Boletín Científico y Técnico INP. 14(5), 40-67

Rossi, R., Mulla, D., Journel, A. y Franz, E. (1992). Geostatistical tolos for modeling and interpreting ecological spatial dependence. Ecological Monographs. Ecological Society of America 62(2), 277-314.

Villon, C. (1995). Description of the artisanal fisheries in Ecuador. A dissertation presented to the University of Aberdeen in partial fulfillment of the requirements for the Degree of Master of Marine and Fisheries Sciences. Dip. Mar. And fish, Sciences. Aberdeen - Scotland. 111 pp.

Wonnacott, R. y Wonnacott, T. (1991). Estadística básica práctica. Usos y aplicaciones. Grupo Noriega. Editores LIMUSA. México. 243-255 pp.

Yáñez-Arancibia, A., Lara-Domínguez, L., Rojas-Galaviz, JL., Sánchez-Gil, P., Day, JW., \& Madden, CJ. (1988). Seasonal biomass and diversity of estuarine fishes coupled with tropical habitat heterogeneity (southern Gulf of México). J. Fish Biol. 33: 191-200. 